\title{
TU/e EmonONEN

\section{Perceived speed of changing color in chroma and hue directions in CIELAB}

\section{Citation for published version (APA):}

Kong, X., Murdoch, M., Vogels, I., Sekulovski, D., \& Heynderickx, I. (2019). Perceived speed of changing color in chroma and hue directions in CIELAB. Journal of the Optical Society of America A, Optics, Image Science and Vision, 36(6), 1022-1032. https://doi.org/10.1364/JOSAA.36.001022

DOI:

10.1364/JOSAA.36.001022

Document status and date:

Published: 01/06/2019

\section{Document Version:}

Publisher's PDF, also known as Version of Record (includes final page, issue and volume numbers)

\section{Please check the document version of this publication:}

- A submitted manuscript is the version of the article upon submission and before peer-review. There can be important differences between the submitted version and the official published version of record. People interested in the research are advised to contact the author for the final version of the publication, or visit the $\mathrm{DOI}$ to the publisher's website.

- The final author version and the galley proof are versions of the publication after peer review.

- The final published version features the final layout of the paper including the volume, issue and page numbers.

Link to publication

\section{General rights}

Copyright and moral rights for the publications made accessible in the public portal are retained by the authors and/or other copyright owners and it is a condition of accessing publications that users recognise and abide by the legal requirements associated with these rights.

- Users may download and print one copy of any publication from the public portal for the purpose of private study or research.

- You may not further distribute the material or use it for any profit-making activity or commercial gain

- You may freely distribute the URL identifying the publication in the public portal.

If the publication is distributed under the terms of Article 25fa of the Dutch Copyright Act, indicated by the "Taverne" license above, please follow below link for the End User Agreement:

www.tue.nl/taverne

Take down policy

If you believe that this document breaches copyright please contact us at:

openaccess@tue.nl

providing details and we will investigate your claim. 


\title{
Perceived speed of changing color in chroma and hue directions in CIELAB
}

\author{
Xiangzhen Kong, ${ }^{1,2}$ (1) Michael J. Murdoch, ${ }^{3, *}$ (1) Ingrid Vogels, ${ }^{2}$ Dragan Sekulovski, ${ }^{4}$ and \\ INGRID HEYNDERICKX ${ }^{2}$ \\ ${ }^{1}$ Department of Industrial Engineering \& Innovation Sciences, Eindhoven University of Technology, Eindhoven 5600MB, The Netherlands \\ ${ }^{2}$ School of Computer Science and Technology, Wuhan University of Technology, Wuhan 430070, China \\ ${ }^{3}$ Munsell Color Science Laboratory, Rochester Institute of Technology, Rochester, New York 14623, USA \\ ${ }^{4}$ Signify Research, High Tech Campus 7, Eindhoven 5656 AE, The Netherlands \\ *Corresponding author: michael.murdoch@mail.rit.edu
}

Received 23 January 2019; revised 8 April 2019; accepted 19 April 2019; posted 19 April 2019 (Doc. ID 358479); published 17 May 2019

\begin{abstract}
In dynamic LED lighting, the perceived speed of changing color is an important concept; however, there exists no suitable temporal color space. In a psychophysical experiment, we compared the perceived speed of periodic temporal transitions in CIELAB chroma and hue directions around five base colors [the five Munsell hues: 5R (red), 5Y (yellow), 5G (green), 5B (blue), and 5P (purple)]. The experiment was conducted in a light laboratory, with the main illumination stimulus subtending a visual angle of $101 \times 77 \mathrm{deg}$. In sequential paired presentations, observers were asked to identify which transition appeared faster, and points of subjective equality between transitions were computed. The speed of transitions was defined in CIELAB $\Delta E_{a b}^{*} / s$, which was shown to be temporally non-uniform; uniformity was improved using a modified color space based on speeds in the DKL
\end{abstract} space of Derrington et al. [J. Physiol. 357(1), 241 (1984)]. @ 2019 Optical Society of America

https://doi.org/10.1364/JOSAA.36.001022

\section{INTRODUCTION}

The development of light-emitting diode (LED) technology has enabled easy and inexpensive ways to create dynamic colored light. As a result, dynamic colored light is nowadays used in several applications, for instance, to enhance the alertness of office workers [1], to create appealing light atmospheres [2-5], and to enhance the immersive experience of displays, such as in the Philips Ambilight TV [6]. In most of these applications, the light has to fulfill (at least) the following two requirements in order to be attractive to observers: the light should change smoothly over time, in luminance and/or chromaticity, and the light should change at the desired rate of change (or speed) $[4,7,8]$. Several studies have investigated how people perceive smoothness of dynamic light generated with limited temporal resolution [7], subtlety of dynamic light [4], and its attractiveness [2]. However, it is not known yet how to describe the perceived rate of a temporal change in color. For instance, if we want to generate a temporal light transition with a constant perceived rate of change, we need to know what is perceived as an equal amount of change in color per time unit.

Color science has long focused on perceptual differences between colors in the spatial domain, leading to metrics such as CIEDE94 and CIEDE2000 that describe the magnitude of a color difference for spatially separated colors. These metrics are formulated in the perceptually uniform CIELAB color space.
Although the uniformity of the CIELAB color space is not perfect (since it depends, among other things, on hue [9]), this color space is considered useful for describing how to make smooth spatial color gradients. Since there is currently no validated model for temporal color perception available, it is most reasonable to use CIELAB to describe temporal color changes as well, and to express the speed of a color transition in terms of $\Delta E_{a b}^{*} / s$. This was done for the first time in 2007 by Sekulovski et al. [7], who measured the maximum color difference between successive colors at which a temporal color transition was still perceived as smooth. When the smoothness threshold was expressed in terms of $\Delta E_{a b}^{*} / s$, a linear relationship was found with the temporal frequency. However, the smoothness threshold was a factor of 10 lower for temporal changes in lightness compared to changes in hue and chroma. This means that the CIELAB color space is quite non-uniform for describing temporal color differences. Therefore, the question remains how to describe the speed of a temporal color transition in a perceptually uniform way.

Existing literature on speed perception cannot be used to answer this question since it manipulates other (for the purpose of our study irrelevant) stimulus characteristics. For example, a few studies have measured the sensitivity to changing luminance for achromatic Ganzfeld stimuli (e.g., [10]). Here, log luminance per min was used as a metric for describing the temporal change in luminance. However, in this study, we are 
interested in the sensitivity to changing chromaticity. In addition, a large amount of literature exists on motion perception for spatiotemporal stimuli, for which speed can be expressed as degrees per second (where "degrees" refer to the spatial distance in terms of visual angle). However, we want to know the perceived speed of a light transition for a (ideally) homogeneous light stimulus. Since the spatial structure of the stimulus is constant, there is no change in visual angle.

Our long-term goal is to develop a universal metric for describing the perceived rate of a temporal color change. In this paper, we aim to present new data on speed perception using temporal stimuli defined in CIELAB and evaluate how well existing color models, both CIELAB and cone spaces including LMS and Derrington, Krauskopf, and Lennie (DKL) [11] describe the results. Therefore, we conducted a psychophysical experiment in which we measured the perceived speed of temporal linear color transitions in chroma and hue at constant lightness in the CIELAB color space. In order to quantify the perceived magnitude of the speed, several stimuli (with different speeds) were compared to a reference stimulus (with a fixed speed), and the point of subjective equality (PSE) in speed was determined. As expected, none of the models just mentioned were suitable for predicting perceived rate of color change. We propose a straightforward adjustment to these models and evaluate which model is the most promising for further improvement and validation.

\section{METHODS}

The experiment was designed to find the PSE of speed between different color transitions. To do so, a two-interval forced-choice task was used. More particularly, participants were instructed to compare the perceived speed of two temporal color transitions (i.e., a reference stimulus and a comparison stimulus). The transitions varied either in CIELAB chroma or hue direction around a base color point. Five base color points were used, corresponding to the principal Munsell hues 5R (red), 5Y (yellow), 5G (green), 5B (blue), and 5P (purple) [12]. We limited ourselves to two types of stimulus pairs: (1) one stimulus was modulated in the chroma direction and the other stimulus in the hue direction around the same base color point, referred to as $\mathrm{CH}$ comparison; (2) the two stimuli were both modulated in the hue direction around two different base color points, referred to as $\mathrm{HH}$-comparison. A third possible type of comparison, namely $C C$-comparison (i.e., two stimuli both modulated in the chroma direction around two different base color points), were omitted in this experimental design to limit the experimental time for the subjects, and because higher-amplitude $C C$ stimuli would have resulted in out-of-gamut colors in some conditions. The method of constant stimuli was used to find the PSE of speed with respect to the reference stimulus by fitting a psychometric function through the percentages "the comparison stimulus is faster than the reference stimulus", averaged over the participants. The experiment used a full-factorial within-subject design with 26 participants.

\section{A. Experimental Setup}

The experiment was conducted in a laboratory (see Fig. 1) that is designed to study dynamic color perception and adaptation,

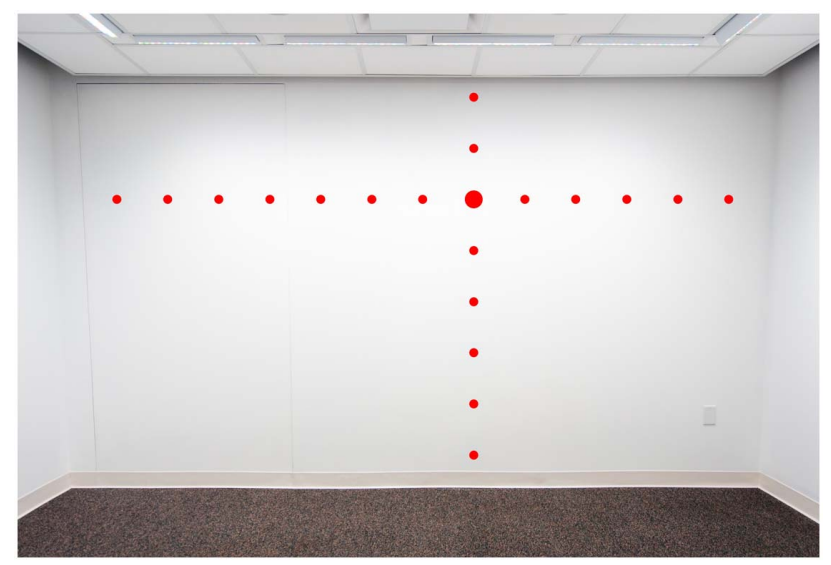

Fig. 1. Photograph of the DVA Lab with a neutral white $4000 \mathrm{~K}$ light setting. The red dots added to the photo indicate the positions on the wall that were measured; the larger dot shows the location of the characterization measurements.

dubbed the Dynamic Visual Adaptation (DVA) Lab of the Munsell Color Science Lab of Rochester Institute of Technology. The DVA Lab is a $3.66 \mathrm{~m} \times 4.27 \mathrm{~m} \times 2.44 \mathrm{~m}$ (length $\times$ depth $\times$ height) room with a variegated gray carpet and four white walls without a window. All the walls are painted with a matte white paint having an average reflectance of $93 \%$, and the floor has an average reflectance of $7 \%$. One of the long walls consists of three sliding wooden panels $2.4 \mathrm{~m}$ wide, which can be opened. The room is equipped with a five-primary (red, green, blue, mint green, white: RGBMW) LED lighting system that can be addressed at $40 \mathrm{~Hz}$ to create smooth temporal changes. The lighting system consists of 14 Philips SkyRibbon wall-washing fixtures that are mounted in the ceiling to illuminate the lab's walls, resulting in a smoothly non-uniform illumination pattern. The uniformity was assessed via spectral measurements at positions spaced $30 \mathrm{~cm}$ apart, noted by red dots in Fig. 1; the larger red dot is the location of the brightest region of the wall, where the spectral characterization data used to model the lighting system were measured. Vertically, the point above and the two points below the reference were all above $70 \%$ of the reference luminance, and the top and bottom points were $38 \%$ and $40 \%$ of the reference. Horizontally, the central nine points (the middle $2.8 \mathrm{~m}$ of the wall) all measured above $86 \%$ of the reference luminance, and the outermost points reached $38 \%$. Chromaticity uniformity is described in Section 2.F below, after the color stimuli are explained. More information about the lab is described elsewhere [13]. We intentionally chose a full room setting instead of the often used $2^{\circ}$ or $10^{\circ}$ stimuli, since dynamics in general lighting are more realistically represented with a larger field of view; similarly, we allowed participants to look freely around the illuminated wall.

\section{B. Stimuli}

The stimuli were periodic temporal color transitions around a base color point in the chroma or hue direction of the CIELAB LCh color space [see Fig. 2(a)]. The color changed every $\Delta t$ seconds with a step size $S$ over a full period of $3 \mathrm{~s}$, which 


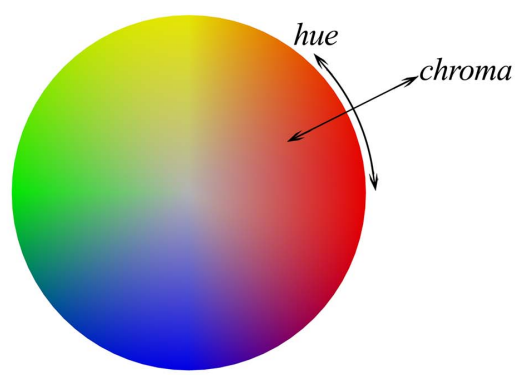

(a)

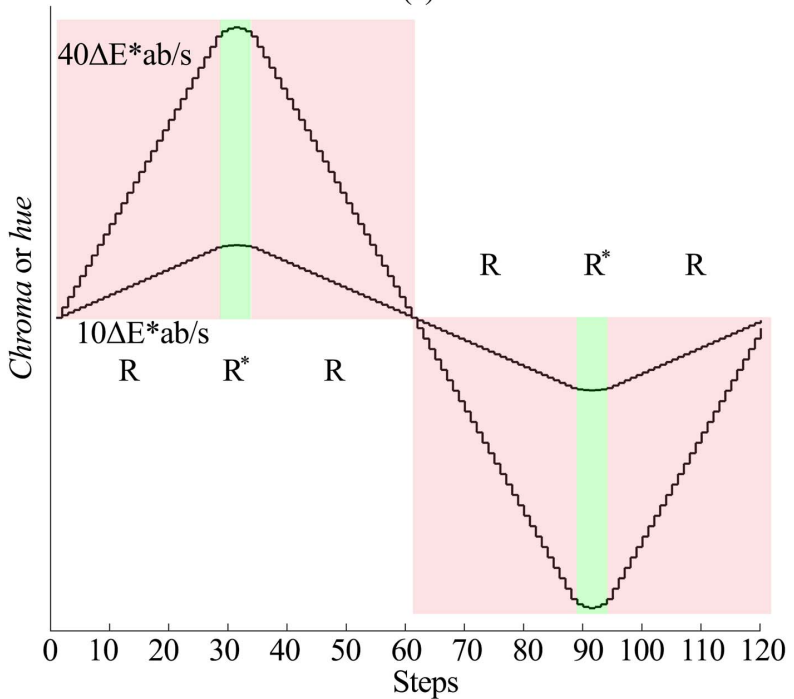

(b)

Fig. 2. (a) Chroma and hue directions in the Ch plane of CIELAB LCh color space. (b) Temporal color transition as a function of time with a speed of $10 \Delta E_{a b}^{*} / s$ or $40 \Delta E_{a b}^{*} / s$. The regions marked $\mathrm{R}$ have a fixed step size, and hence a fixed speed. The region marked $\mathrm{R}^{*}$ is adapted to smooth the abrupt change in color.

was found in pilot experiments to be both long enough to be visibly changing and short enough to allow many stimulus presentations in a manageable experiment length. The color transition was shown as five full periods of a triangular wave to allow for easier judgements on the speed of the transition. The sharp edges of the triangular wave were smoothed, as indicated by the regions $\mathrm{R}^{*}$ in Fig. 2(b). This was done in order to avoid abrupt changes that might influence the perception of speed, as suggested in [7].

Each cycle had a fixed number of discrete steps $(\mathrm{N}=120)$, and each step was shown for a fixed period $\Delta \mathrm{t}=0.025 \mathrm{sec}-$ onds. Thus, the stimulus lasted for 15 (i.e., $5 \times 120 \times 0.025$ ) seconds. $10 \%$ (i.e., $\mathrm{p}=0.1$ ) of this stimulus duration was used for smoothing the abrupt color change. Hence, the stimulus consisted of $\mathrm{N} \times(1-\mathrm{p})$ number of steps with a fixed step size $S$ and $\mathrm{N} \times \mathrm{p}$ steps with variable step size $S^{*}=\frac{S}{2^{i}}(1 \leq \mathrm{i} \leq \mathrm{N} \times \mathrm{p})$. The color transition speed (CTS) is defined as the speed of the linear part of the transition and is calculated as

$$
\mathrm{CTS}=\mathrm{S} / \Delta \mathrm{t} \approx \mathrm{A} / \mathrm{T},
$$

with $\mathrm{A}$ being the amplitude of the transition and $\mathrm{T}$ the duration of one half of the cycle (i.e., $1.5 \mathrm{~s}$ ). In our experimental design, the duration of the transition was fixed, and the speed of the color transition was implemented by changing the amplitude of the transition [see Fig. 2(b)]. The amplitude A can be calculated as follows:

$$
A=S \times\left[N \times(1-p)+N \times p \times\left(1-\frac{1}{2^{\mathrm{N} \times \mathrm{p}}}\right)\right] .
$$

Each color transition varied around a base color point, which corresponded to a principal Munsell hue: 5R (red), 5Y (yellow), 5G (green), 5B (blue), or 5P (purple). The color points of the principal Munsell hues were calculated as follows: first, $10^{\circ}$ $\mathrm{XYZ}$ tristimulus values were computed from the reflectance spectra [14] of the matte Munsell color chips with the principal hues under Illuminant $\mathrm{C}$. Because Munsell colors are defined to be uniform under Illuminant C, Von-Kries chromatic adaptation was used to find the corresponding color points under $4000 \mathrm{~K}$, which was the white point of the neutral light setting used in the experiment. These XYZ tristimulus values were transformed to CIELAB LCh, with the adaptation white of $4000 \mathrm{~K}$ at a wall luminance level of $200 \mathrm{~cd} / \mathrm{m}^{2}$, and the average CIELAB $h$ for each group of Munsell hues was found. Each of the five base color points was defined at its average CIELAB hue with a chroma $C^{*}$ of 60 and a lightness $L^{*}$ of 90 . The corresponding CIELAB hue angles are 27 for $5 \mathrm{R}, 18$ for $5 \mathrm{Y}, 159$ for $5 \mathrm{G}, 221$ for $5 \mathrm{~B}$, and 302 for $5 \mathrm{P}$.

Each color transition varied around the base color point at a constant speed in either the chroma or hue direction. Five color transition speeds were used: $2.5,5,10,20$, and $40 \Delta E_{a b}^{*} / s$. Since the transitions were described in the CIELAB LCh color space, first the corresponding speeds in terms of chroma and hue were calculated, using

$$
\begin{gathered}
\Delta C^{*}=\Delta E_{a b}^{*}, \\
\Delta H^{*}=\arccos \left(1-\frac{\Delta E_{a b}^{* 2}}{2 \times C^{* 2}}\right) .
\end{gathered}
$$

Then the $L^{*}, C^{*}$, and $h$ values were transformed to $\mathrm{XYZ}$ values, and finally to the RGBW values that were used to control the LED luminaires. The $X Y Z$ to $R G B W$ transformation model that was used, with a white mixing ratio of 0.85 , has been described in more detail in [13].

All the stimuli were within the gamut of the system. During the experiment, all the walls were illuminated; however, the seated participants were asked to look at the wall in front of them.

\section{Experimental Conditions}

In each trial, two color transitions were shown sequentially, in random presentation order: a reference stimulus and a comparison stimulus. In $\mathrm{CH}$-comparisons, the reference stimulus was modulated in the hue direction around one of the five base color points at a speed of $10 \Delta E_{a b}^{*} / s$, whereas the comparison stimulus varied around the same base color point in the chroma direction at one of the five speeds. Hence, there were 25 (i.e., 5 hue $\times 5$ speed) $\mathrm{CH}$-comparisons. In $H H$-comparisons, both stimuli were modulated in the hue direction. The reference stimulus varied around a hue of $5 \mathrm{R}$ (i.e., $h=27^{\circ}$ ) at a speed of $10 \Delta E_{a b}^{*} / s$, and the comparison stimulus varied around one of the five base color points at one of the five speeds. 
This means that there were also 25 (i.e., 5 hue $\times 5$ speed) $H H$ comparisons.

\section{Participants}

Twenty-six adults (15 males and 11 females) volunteered to participate in the experiment. Their age ranged from 21 to 53 years $($ mean $=32.4$, standard deviation $=10.5)$. All participants had normal color vision, as measured with the Ishihara test for color deficiency. Fourteen of them were wearing glasses, and six were wearing contact lenses. Most participants had more-than-average knowledge about color, while three of them had advanced knowledge about color. Ten participants did not have any experience with lighting experiments, while the others had different levels of experience.

\section{E. Procedure}

The experiment was approved by the Institutional Review Board at RIT, the Human Subject Research Office. Before the start of the experiment, participants were asked to read and sign an informed consent form, in which they confirmed their voluntary participation.

When participants entered the experimental room, the light was set to a static white light with a correlated color temperature of $4000 \mathrm{~K}$ and a wall luminance of $200 \mathrm{~cd} / \mathrm{m}^{2}$. Participants were instructed to sit on a chair that was positioned at a distance of $1.5 \mathrm{~m}$ from the wall in front of them. The average eyesight level was about $1.5 \mathrm{~m}$ above the floor. No chin or forehead rest was used since we intended to have a more natural viewing setting. Then the experimenter read the instruction script that consisted of the following parts: welcome words, explanation of the necessity to do an Ishihara test, collection of demographic information, explanation of the stimuli and task, illustration of the voice instructions, and a practice session. The participants were instructed that they were free to make head and eye movements, as long as they looked at the wall in front of them. During the practice session, each observer was asked to compare (1) the speeds of a hue change with $10 \Delta E_{a b}^{*} / s$ at $5 \mathrm{R}$ and a chroma change with $40 \Delta E_{a b}^{*} / s$ at $5 \mathrm{R}$, and (2) the speeds of a hue change with $10 \Delta E_{a b}^{*} / s$ at $5 \mathrm{R}$ and a hue change with $2.5 \Delta E_{a b}^{*} / s$ at $5 \mathrm{G}$. Each comparison was repeated twice. Six computer-generated voice instructions were used: V1, "First stimulus"; V2, "Second stimulus"; V3, "Which stimulus appears faster?"; V4, "Input recorded"; V5, "Invalid input, please input again"; V6, "Congratulations! You finished the experiment, thank you for your participation".

The flow of presenting the stimulus pairs is shown in Fig. 3, and consisted of voice-cued sequential stimuli interspersed with neutral $4000 \mathrm{~K}$ lighting that was shown for ten seconds. Research has shown that after ten seconds, chromatic adaptation is approximately $80 \%$ complete [15]. This means that participants started from approximately the same adaptation point.

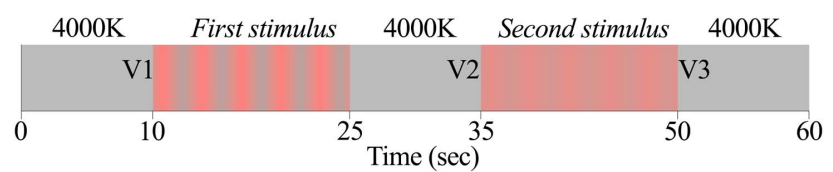

Fig. 3. Illustration of the procedure for presenting one stimulus pair. V1, V2, and $\mathbf{V} 3$ are the voice instructions.
Participants were instructed to judge which of the two sequential color transitions appeared to be faster by pressing a button on a keyboard: " 1 " to indicate the first stimulus appeared faster and " 2 " to indicate the second stimulus appeared faster. If a participant happened to press a button that was neither "1" nor " 2 ", the voice instruction $\mathbf{V} 5$ would be played until that participant pressed "1" or "2". Then V4 would be played and the program would proceed. Since participants had different levels of knowledge about color, the CIELAB LCh space was briefly explained, and it was pointed out that all the stimuli were only changing in either the chroma or hue direction at a constant luminance level. In addition, we emphasized that all stimuli had the same duration, and that a judgement regarding the speed of the transition could be made based on the slope of the transition. The concept of slope was explained by showing a similar figure to Fig. 2(b). All the stimuli shown during the instruction and practice phase were part of the stimuli of the main experiment. Participants were allowed to ask questions during the instructions and they had the opportunity to repeat the practice session, if needed, before the start of the main experiment. During the practice session and the main experiment, participants wore a baseball cap to prevent them from looking directly into the luminaires mounted in the ceiling.

The main experiment consisted of two sessions. In the first session, participants evaluated all 50 stimulus pairs [i.e., 25 $\mathrm{CH}$-comparisons (5 hue $\times 5$ speed) $+25 \mathrm{HH}$-comparisons ( 5 hue $\times 5$ speed)]. In the second session, only the three intermediate speeds were presented, resulting in 30 comparisons [i.e., $15 \mathrm{CH}$-comparisons $(5$ hue $\times 3$ speed $)+15 \mathrm{HH}$ comparisons (5 hue $\times 3$ speed)]. As a result, the largest and smallest speeds of the comparison stimulus were presented only once, while the intermediate three speeds were repeated twice per participant. We decided to do this since the lowest and highest speed were visually quite different from the reference speed, and they were basically used (1) to test if participants understood the task by checking whether their responses were close to $100 \%$ correct, and (2) to make participants feel more confident about their performance by showing some easier trials. In each session, first all $\mathrm{CH}$-comparisons were presented, blocked per base color, and then all $H H$-comparisons were shown. The presentation order of the base colors was based on a balanced Latin square design [16].

The second session was arranged at least half an hour after the first session. For some participants, the sessions took place on different days. All participants watched the demo stimuli and did the practice session again before the start of the second session in order to be sure that they had the same level of understanding. After each session, participants were asked to answer the questions shown in Table 1. They were encouraged to elaborate on their answers, and not simply respond with "yes" or "no".

\section{F. Verification Measurements}

All stimuli were verified (i.e., each step in the temporal color transition was measured) with a Photo Research PR-655 spectroradiometer. The location of the measurement was at $1.7 \mathrm{~m}$ above the floor, as indicated by the larger red circle in Fig. 1. Among the 6000 (i.e., $50 \times 120$ ) measured steps, the average 
Table 1. Questionnaire after Each Session

\begin{tabular}{lcl}
\hline Session & No. & \multicolumn{1}{c}{ Question } \\
\hline 1 & 1 & $\begin{array}{l}\text { Do you think the task of the experiment is } \\
\text { difficult? If so, what made it difficult? } \\
\text { Where did you see the most visible color change? } \\
\text { "Center", "Periphery", Or "No difference } \\
\text { (between center and periphery)?" }\end{array}$ \\
3 & $\begin{array}{l}\text { Did you notice anything that was not smooth } \\
\text { (such as stepping, flickering, stuttering, etc.)? If so, } \\
\text { please describe it (e.g., in which color). } \\
\text { Do you feel the cap is influencing the judgement? }\end{array}$ \\
\hline 2 & 5 & $\begin{array}{l}\text { Do you feel the experiment is easier the second } \\
\text { time than the first time? } \\
\text { Any other comments? }\end{array}$ \\
\hline
\end{tabular}

deviation from the corresponding intended colors was 2.34 $\Delta E_{a b}^{*}$, with the largest deviation being $4.81 \Delta E_{a b}^{*}$. From the measurements, the actual transition speeds that were presented to the observers were further calculated, and the results showed that the speeds differed from the intended speeds by $2.17 \%$.

The spatial chromaticity uniformity of the wall was measured at the same spatial locations shown in Fig. 1, and Euclidean $\Delta u^{\prime} v^{\prime}$ values from the reference point were computed. Over all five base colors and the neutral white, at all spatial locations (total $6 \times 19$ measurements), the median $\Delta u^{\prime} v^{\prime}$ from the reference was 0.0010 . The maximum difference was 0.0042 for the $5 \mathrm{Y}$ base color near the bottom of the wall.

\section{RESULTS}

\section{A. Calculation of PSEs}

First, we checked the reliability of the participant responses. In either $\mathrm{CH}$ - or $\mathrm{HH}$-comparisons, the extreme speeds (i.e., 2.5 and $\left.40 \Delta E_{a b}^{*} / s\right)$ were visually quite different from the reference speed of $10 \Delta E_{a b}^{*} / s$. However, in 29 of the 520 cases [i.e., 26 participants $\times 2$ speeds $\times 2$ types of comparisons $(\mathrm{CH}$ and $H H) \times 5$ hues], participants either reported that the lowest speed was faster than the reference or the highest speed was slower than the reference. In particular, there was one participant with 6 out of 20 likely "incorrect" responses. Therefore, the data of this participant was removed for further analysis.

For each comparison type ( $\mathrm{CH}$ or $\mathrm{HH})$ and base color, the percentage of responses "the comparison stimulus is faster than the reference stimulus" was calculated over all participants and plotted as a function of the speed of the comparison stimulus. This resulted in 10 plots. For $\mathrm{CH}$ comparisons the reference was a transition of $10 \Delta E_{a b}^{*} / s$ along the hue direction around the same hue as the comparison stimulus. For $H H$ comparisons, the reference was a transition of $10 \Delta E_{a b}^{*} / s$ along the hue direction around a (fixed) hue of 5R.

The resulting data were fitted with the psychometric function of Eq. (5), using a generalized linear model (i.e., the glmfit function available from the Statistics Toolbox in MATLAB 2017b):

$$
f=\frac{1}{1+e^{-\left(a+b \log _{10}(x)\right)}} \times 100 \%
$$

In this equation, $f$ is the percentage of responses, $x$ corresponds to the speed, the parameter $a$ defines the percentage when $x=0$, and the parameter $b$ is related to the slope of the psychometric function. The PSE is defined as the speed at which the percentage of responses "faster than the reference" equals $50 \%$.

Figure 4 shows the ten psychometric curves fitted with the data of the 25 participants. In this figure, the speed is plotted on a logarithmic scale to improve the goodness of fit. The output of the logarithmic fit has a root mean square error of 1.68 $\Delta E_{a b}^{*} / s$, compared to the root mean square error of 2.97 $\Delta E_{a b}^{*} / s$ for the linear fit. We used a statistical technique based on Monte Carlo resampling [17] to estimate the variability in the parameters $a$ and $b$ of the psychometric function. To do so, we adopted a non-parametric bootstrap by treating all data points of all participants (for a given condition, i.e., $\mathrm{CH}$ or $\mathrm{HH}$ comparison and one base color) as if they reflected one underlying distribution. Next, 25 new data points were randomly chosen from this distribution for each of the five speeds and the PSE was calculated. This procedure was repeated 10,000 times, and from these data we calculated the average PSE and its confidence interval as the 2.5th and 97.5th percentile. These data are summarized in Fig. 5.

The straightforward interpretation of the PSE is that, for example, a transition of $18.48 \Delta E_{a b}^{*} / s$ along the chroma direction at $5 \mathrm{Y}$ is perceived as fast as a transition of $10 \Delta E_{a b}^{*} / s$ along the hue direction at $5 \mathrm{Y}$ [Fig. 5(a)]. At the same time, a transition of $6.12 \Delta E_{a b}^{*} / s$ along the hue direction at $5 \mathrm{Y}$ is perceived as fast as a transition of $10 \Delta E_{a b}^{*} / s$ along the hue direction at 5R [Fig. 5(b)]. To describe the data in a more compact way, we use the following notation:

$$
\text { PSEs }=\left|\begin{array}{cc}
7.76 & 11.25 \\
18.48 & 6.12 \\
13.52 & 9.30 \\
11.13 & 13.39 \\
19.70 & 5.61
\end{array}\right|
$$

where each row represents a base color (in the order of $5 \mathrm{R}, 5 \mathrm{Y}$, $5 \mathrm{G}, 5 \mathrm{~B}$, and $5 \mathrm{P}$ ), while the first column represents the $\mathrm{CH}$ comparisons and the second column the $H H$ comparisons. From Fig. 5, we can conclude that for the $\mathrm{CH}$ comparisons, the PSE of $5 \mathrm{~B}$ is not significantly different from the reference speed of $10 \Delta E_{a b}^{*} / s$, which basically means that the change in chroma and the change in hue around $5 \mathrm{~B}$ are perceived as equally fast for the same speed in terms of $\Delta E_{a b}^{*} / s$. At the other base colors, the PSE is significantly different from $10 \Delta E_{a b}^{*} / s$, which means that the actual speed of the change in chroma needs to be different (in terms of $\Delta E_{a b}^{*} / s$ ) from the reference speed of the change in hue in order to let them appear equally fast. Similarly, for the $H H$ comparisons, only the PSEs at 5R and $5 \mathrm{G}$ are not significantly different from the reference speed, while the others are. For the PSE at 5R, this is not surprising. Actually, the confirmation that a change in hue with a speed of $10 \Delta E_{a b}^{*} / s$ at $5 \mathrm{R}$ is perceived as equally fast as a change in hue with a speed of $10 \Delta E_{a b}^{*} / s$ at $5 \mathrm{R}$ confirms the reliability of our method. 


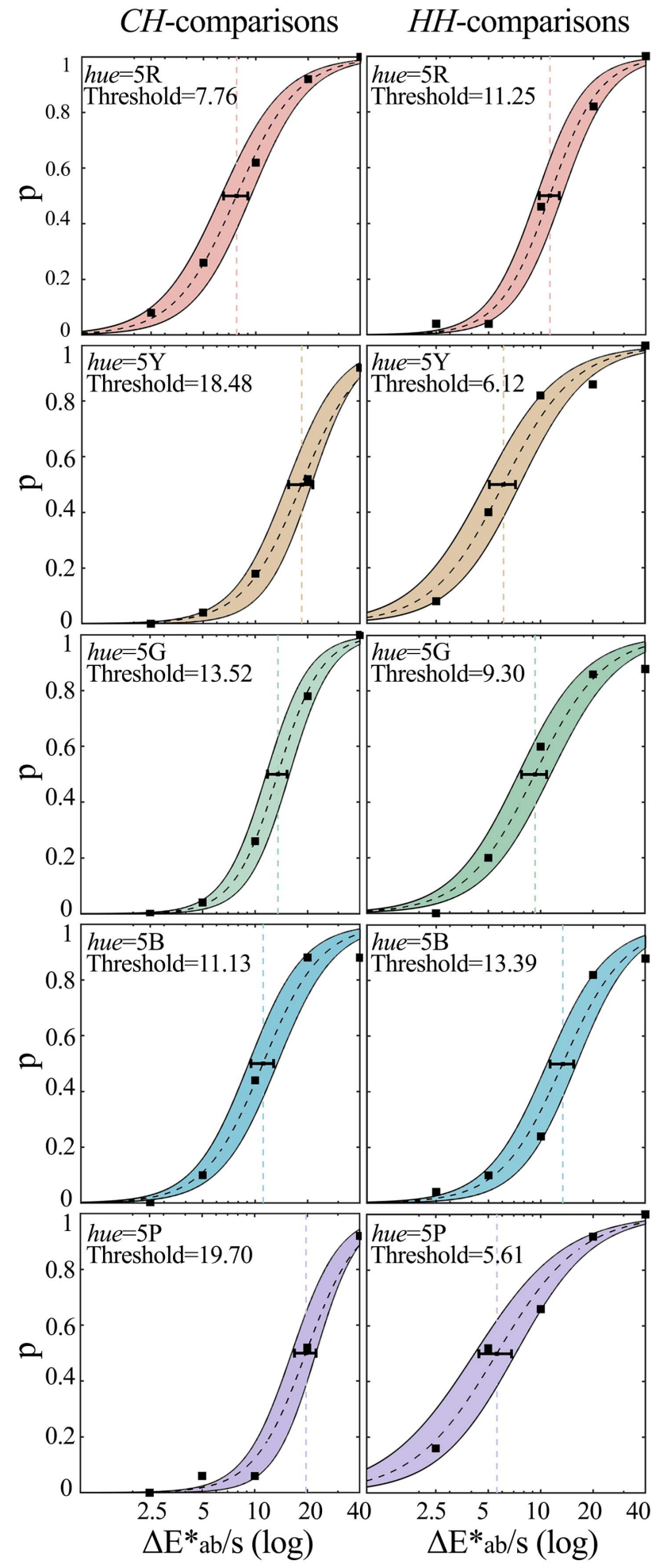

Fig. 4. Proportion of "the comparison stimulus is faster than the reference stimulus" responses against the transition speed of the comparison stimulus for each of the five hues in the $\mathrm{CH}$ comparisons (left column) and the $H H$ comparisons (right column). The filled squares represent the raw percentages. The colored area represents the $95 \%$ psychometric functions, $m$ with the error bars indicating the bootstrap-based $95 \%$ confidence intervals.

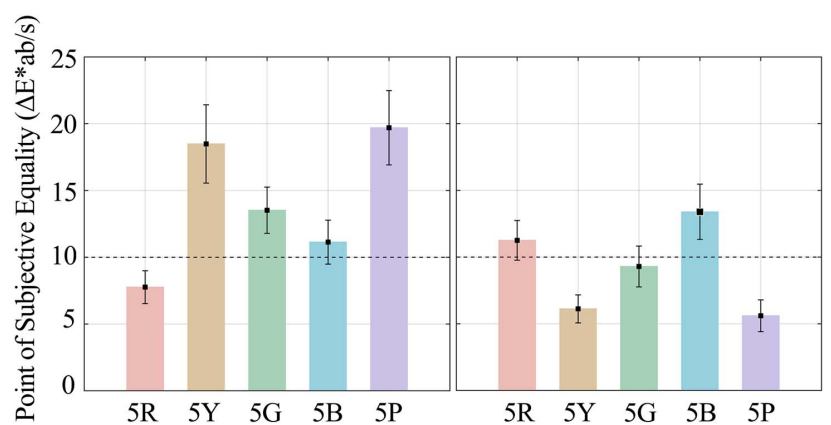

(a)

(b)

Fig. 5. Bar chart of the 10 PSEs with their 95\% CI for (a) $\mathrm{CH}$ comparisons and (b) $H H$ comparisons.

\section{B. Comparison Between 10 PSEs}

When the color transitions would be assessed against the same reference stimulus, their PSE could be directly compared. This was, for example the case for the $H H$ comparisons, the PSEs of which could be mutually compared. To relate the PSEs of all color transitions, we expressed them against the same reference stimulus, using the following two assumptions:

(1) If speed $S_{a}$ for transition A and speed $S_{b}$ for transition B are perceived as equal, then transitions with speeds $\alpha S_{a}$ and $\alpha S_{b}$ are perceived equal as well (law of proportionality).

(2) If the perceived speed of transition A and transition B are equal, and the perceived speed of transition $\mathrm{B}$ and transition $\mathrm{C}$ are equal, then the perceived speed of $\mathrm{A}$ and $\mathrm{C}$ are also equal (law of transitivity).

Based on these two assumptions, we related the speed of all color transitions to the same reference, being a speed of 10 $\Delta E_{a b}^{*} / s$ in the hue direction around $5 \mathrm{R}$. From the notation in Eq. (6), we know that a transition speed of $10 \Delta E_{a b}^{*} / s$ along the hue direction at $5 \mathrm{Y}$ was perceived as fast as a transition speed of $(10 / 6.12) \times 10=16.34 \Delta E_{a b}^{*} / s$ along the hue direction at $5 \mathrm{R}$, and as fast as a transition speed of $18.48 \Delta E_{a b}^{*} / s$ along the chroma direction at $5 \mathrm{Y}$. Thus, to compare the transition speed along the chroma direction at $5 \mathrm{Y}$ with a transition speed along the hue direction at $5 \mathrm{R}$, we need to divide 18.48 by $(10 / 6.12)$; as a result, a transition speed of $11.31 \Delta E_{a b}^{*} / s$ along the chroma direction at $5 \mathrm{R}$ is assumed to be perceived as fast as a transition speed of $10 \Delta E_{a b}^{*} / s$ along the hue direction at $5 \mathrm{R}$. Similarly, all the PSE values could be related to the same reference stimulus of a transition speed of $10 \Delta E_{a b}^{*} / s$ along the hue direction at $5 R$, which results in

$$
\text { PSEs }=\left|\begin{array}{cc}
7.76 /(10 / 11.25) & 11.25 \\
18.48 /(10 / 6.12) & 6.12 \\
13.52 /(10 / 9.30) & 9.30 \\
11.13 /(10 / 13.39) & 13.39 \\
19.70 /(10 / 5.61) & 5.61
\end{array}\right|=\left|\begin{array}{cc}
8.73 & 11.25 \\
11.31 & 6.12 \\
12.57 & 9.30 \\
14.90 & 13.39 \\
11.05 & 5.61
\end{array}\right| .
$$

The PSE values mentioned before can now be directly compared. A value larger than 10 means that the comparison stimulus needs to have a larger speed in order to be perceived equally fast as the reference. Similarly, a value smaller than 10 means 


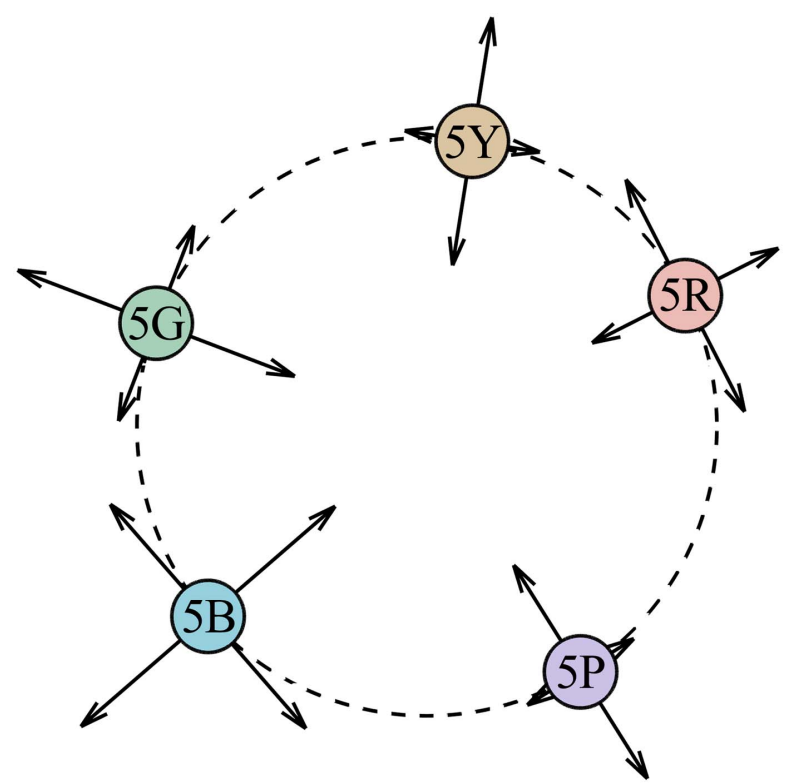

Fig. 6. Length of the arrows in the figure indicates the PSE of the corresponding color transition to make it equally fast as the reference, being a transition speed of $10 \Delta E_{a b}^{*} / s$ along the hue direction around the base color $5 \mathrm{R}$ (represented by length " 1 " in this figure).

that the comparison stimulus needs to have a smaller speed in order to be perceived equally fast as the reference. These relative speeds are depicted in Fig. 6 for the five base color points and the two directions (in CIELAB) used in the experiment. The length of the arrow in Fig. 6 represents how the transition speed in $\Delta E_{a b}^{*} / s$ should be "scaled" in order to make that transition equally fast as the others.
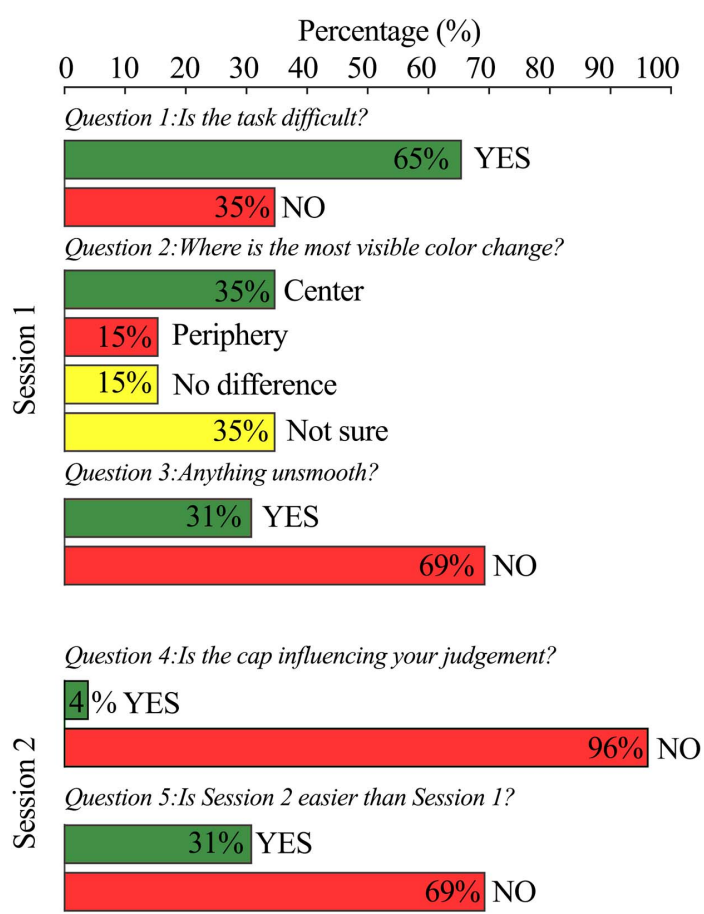

Fig. 7. Overview of the answers given to the questions at the end of Sessions 1 and 2 .

\section{Questionnaire Analysis}

The answers to the questions we asked at the end of Session 1 and 2 are summarized in Fig. 7. 65\% of the participants indicated that the experiment was difficult (Question 1). They mentioned various reasons, among which the lack of a fixation point to help them focus, and the fact that it was hard to memorize speeds. Only $15 \%$ of the participants indicated explicitly that the color change was most visible in their periphery. Some participants $(35 \%)$ reported that some color transitions were unsmooth at some hue values. All participants except one mentioned that the cap did not influence their judgement; instead, they reported that the cap indeed helped to stop them from looking at the luminaires in the ceiling. Some participants $(31 \%)$ reported that Session 2 was more difficult because the speed differences were smaller compared to the stimuli in Session 1.

\section{COMPARISON WITH AVAILABLE COLOR MODELS}

The results presented in Fig. 5 show that color transitions with the same constant speed in CIELAB are not always perceived as having the same speed. This is no surprise, but it confirms that CIELAB cannot be considered a uniform space to describe the perceived speed of color transitions in the chroma and hue directions. An ideal uniform temporal color model should have equal PSE values independent of the base color and modulation direction of the color transition. A possible way of looking at the uniformity of a color model is to check the standard deviation in PSEs, which is zero in the ideal case.

\section{A. Calculation of Slopes in CIELAB}

Despite our assumption that CIELAB would not be temporally uniform, we chose constant $-\Delta E_{a b}^{*} / s$ speeds for this experiment as a starting point. Figure 6 already shows how non-uniform CIELAB is for temporal color transitions, and based on these results we made an attempt to improve CIELAB's uniformity in this respect. This attempt consisted of including a scaling factor in the mutual weighting of $\Delta a^{*} / s$ and $\Delta b^{*} / s$ in the calculation of $\Delta E_{a b}^{*} / s$ as follows:

$$
\Delta E_{a b_{\text {imp }}}^{*} / s=\sqrt{\left(\Delta a^{*} / s\right)^{2}+\alpha\left(\Delta b^{*} / s\right)^{2}},
$$

where $\Delta E_{a b_{\text {imp }}}^{*} / s$ indicates the improved version of $\Delta E_{a b}^{*} / s$. Due to the triangular shape of the stimuli, a linear fit was performed on the three segments of each temporal transition for the trajectory of $a^{*}$ and $b^{*}$ separately. The final $\Delta a^{*} / s$ and $\Delta b^{*} / s$ were calculated using the equation

$$
\text { Slope }=\left(\text { Slope }_{1}-\text { Slope }_{2}+\text { Slope }_{3}\right) / 3 \text {, }
$$

where Slope ${ }_{1}$ covered steps 1-30 of the transition, Slope 2 steps 31-90, and $\mathrm{Slope}_{3}$ steps 91-120. Figure 8 illustrates how $\Delta a^{*} / s$ and $\Delta b^{*} / s$ were obtained.

Based on these slopes, we computed the standard deviation of the PSEs for different values of the weighting factor $\alpha$. Figure 9 shows that this standard deviation is minimal for a value of $\alpha=0.404$. 


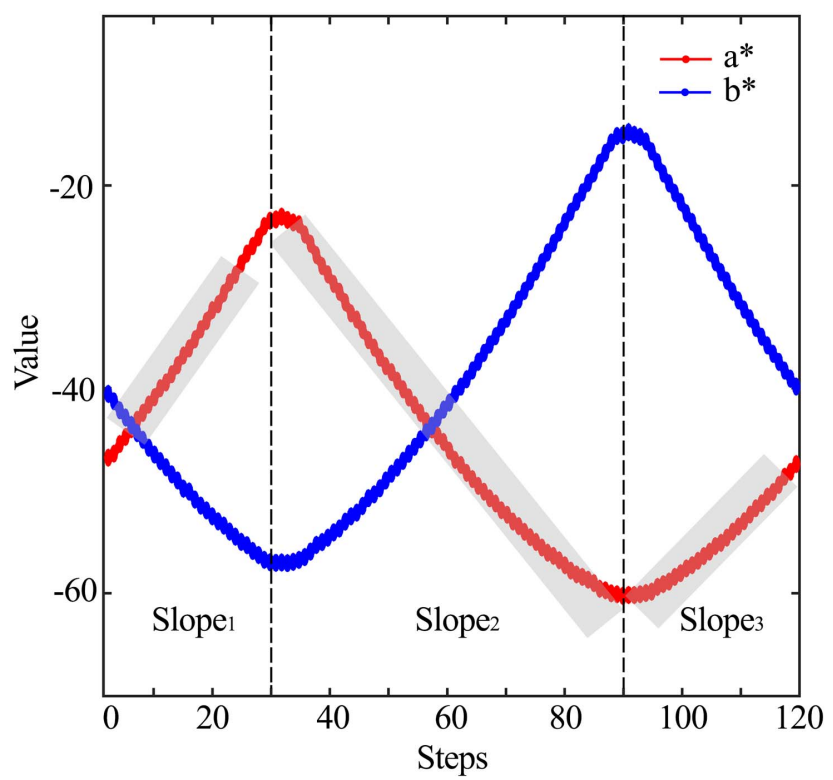

Fig. 8. Illustration of the segments used for calculating the slope of the $a^{*}$ and $b^{*}$ values of the temporal color transition ( $L^{*}$ is constant). Slope $_{1}$ covers the steps $1-30$, while Slope ${ }_{2}$ and Slope $e_{3}$ cover the steps 31-90 and 91-120, respectively. These segments of steps are shown for the change in $a^{*}$ values.

\section{B. Calculation of Slopes in LMS Cone Fundamentals}

We designed our dynamic transitions as constant-slope $C^{*}$ and $h$ changes, which do not correspond to constant-slope $L, M$, and $S$ changes. To calculate the corresponding $\mathrm{L}, \mathrm{M}$, and $S$ cone responses, we used the cone fundamentals of Stockman and Sharpe [18] and the measured spectral data. To minimize the effect of measurement noise, the slopes of $\Delta L / s, \Delta M / s$, and $\Delta S / s$ were calculated in a similar way as shown by Eq. (9). Since neutral white light of $4000 \mathrm{~K}$ was regularly shown before, during, and after the stimuli in the experiment, it was chosen as the adaptation condition; in reality, we expect that observers'

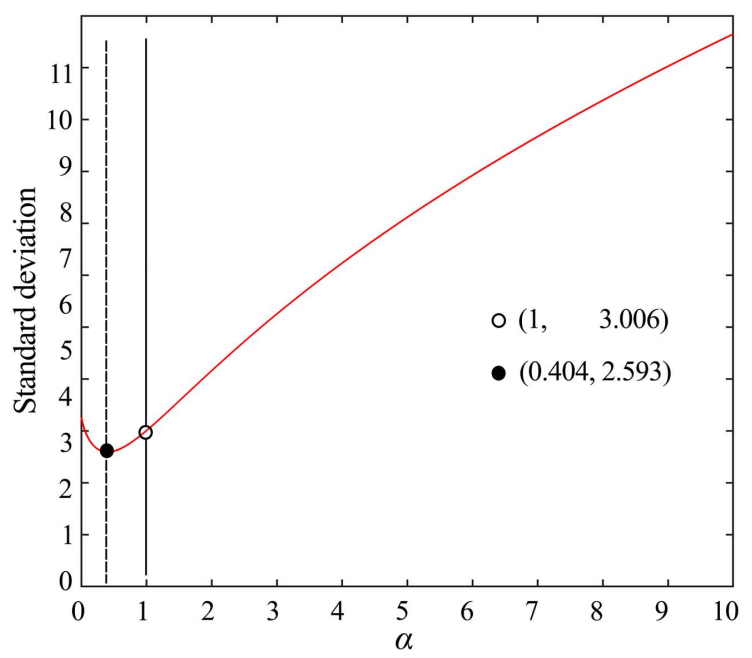

Fig. 9. Standard deviation of PSEs as a function of $\alpha$ in Eq. (8). The open circle represents the standard deviation of CIELAB, while the filled circle is the minima found when $\alpha=0.404$.

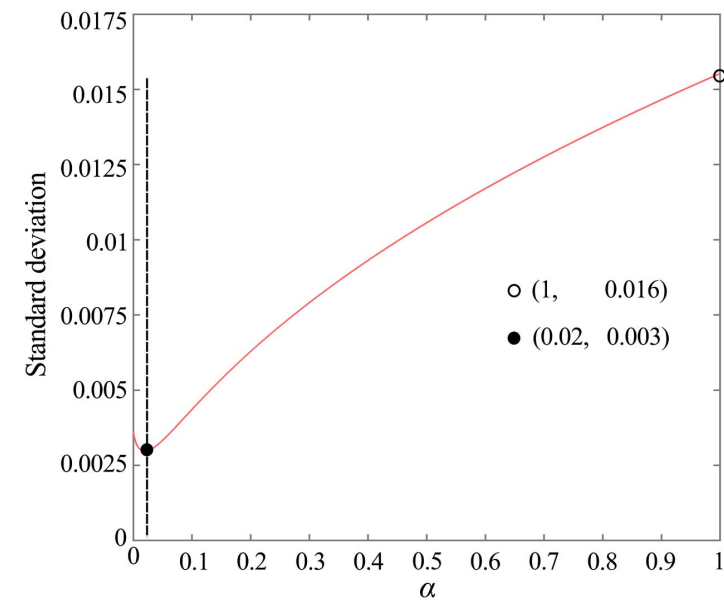

Fig. 10. Standard deviation of PSEs as a function of $\alpha$ in Eq. (11). The open circle represents the standard deviation of DKL, while the filled circle is the minima found when $\alpha=0.02$.

state of adaptation was influenced by the dynamic stimuli, but we don't have a way to predict this variation. Thus, the $L, M$, and $S$ values at each step were normalized by dividing them by the $L, M$, and $S$ values of $4000 \mathrm{~K}$, which is equal to multiplying by $3.28,4.11$, and 11.22 , respectively. However, this normalization step does not influence the calculation of slopes.

Using the same concept of minimizing the standard deviation of PSEs, a scaled LMS space with parameters $\alpha$ and $\beta$ could be constructed as follows:

$$
\Delta L M S / s=\sqrt{(\Delta L / s)^{2}+\alpha(\Delta M / s)^{2}+\beta(\Delta S / s)^{2}} .
$$

The minimum in standard deviation was found when $\alpha$ and $\beta$ were both zero, which means that the $M$ and $S$ responses were eliminated. Eliminating these responses may then give the best fit, but it is intuitively unsatisfying. Thus, based on the physiologically based DKL space [11], which has proven its value in the literature, the following equation was constructed to minimize the standard deviation of the PSEs:

$$
\Delta D K L=\sqrt{[\Delta(L-M) / s]^{2}+\alpha\left\{\Delta[(S-(L+M)] / s\}^{2}\right.} .
$$

Since the $L+M$ channel usually represents luminance, which in our case remains constant, the slope of the $L+M$ channel is ideally zero. Using the same technique as described previously, we now found a minimum for the standard deviation in PSE when $\alpha=0.02$ (as shown in Fig. 10).

\section{DISCUSSION}

\section{A. Optimization of Color Spaces}

Our results demonstrate that more uniformity can be achieved by improving available color spaces. Specifically, the CIELABbased improvement suggests that $\Delta b^{*} / s$ contributes less to speed perception than $\Delta a^{*} / s$, but the resulting reduction in the standard deviation of the PSEs is very small. The improved DKL-based space suggests that $\Delta(S-(L+M) / s$ hardly con- 
tributes to speed perception compared to $\Delta(L-M) / s$. In this way, the standard deviation in PSEs is reduced by almost a factor of 6 . It is important to notice that the small weight $(\alpha=0.02)$ for the $S-(L+M)$ channel may suggest that the $L-M$ channel dominates the speed perception of chromatic change. Although there is no literature for the direct comparison with our results, several papers indicated the "weak" contribution of the $S-(L+M)$ channel in speed perception. For example, Dougherty et al. [19] proposed an equation that combines the luminance, red-green, and blue-yellow opponent mechanisms to describe the mechanism of moving color responses. They assigned the largest weight to the luminance channel $(L+M)$, followed by the weight in the redgreen $(L-M)$ channel and then the blue-yellow $(S-(L+M))$ channel. Nguyen-Tri and Faubert [20] performed a speed-matching experiment using drifting chromatic gratings. They found that the $S-(L+M)$ post-receptoral mechanism does not appear to contribute significantly to determining the perceived speed of chromatic motion. McKeefry and Burton [21], however, did not agree with the opinion that $\mathrm{S}$-cone signals are not as effective in signaling stimulus motion. Their results suggest that the perception of speed is based on $L-M$ and $S-(L+M)$ cone opponent processing.

Figure 11 shows the bar charts of the 10 PSEs in the four spaces: CIELAB and improved CIELAB [Fig. 11(a)], DKL and improved DKL [Fig. 11(b)]. It is not intuitively meaningful to compare these two metrics, as they are of different magnitude. The fact that the optimized DKL space has reduced the standard deviation of PSEs to a larger extent than optimized CIELAB might indicate that DKL is more promising to work with in future. However, that this space implies that the $\mathrm{S}-(\mathrm{L}+\mathrm{M})$ responses can be largely neglected for speed perception of color transitions remains worrisome. It might as well be too simplistic to just target the minimum of the standard deviation of PSEs to improve the uniformity of a color space for temporal transitions. Hence, to gain more insight into how to build a color space with global uniformity for speed perception of temporal color transitions, more data collection and more extensive modeling are needed.
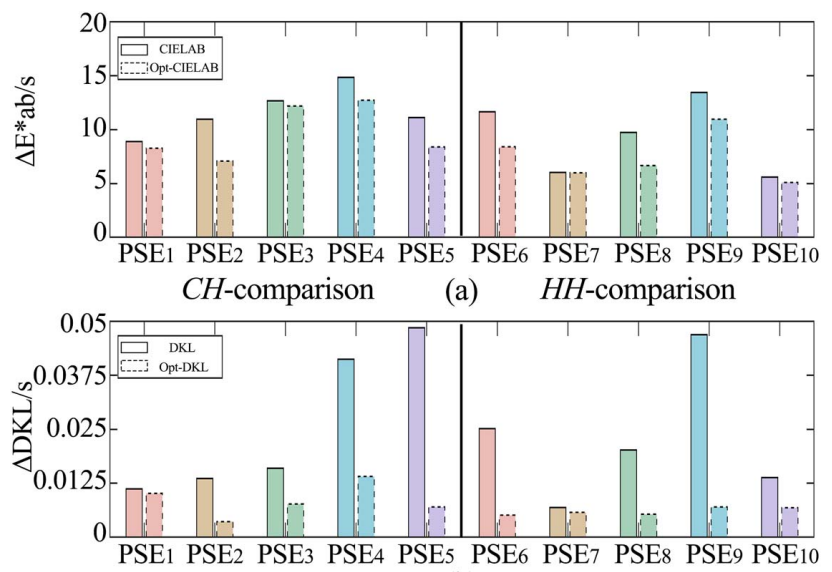

(b)

Fig. 11. Bar charts of the 10 PSEs expressed in different color spaces: (a) CIELAB and improved CIELAB; (b) DKL and improved DKL.

\section{B. Limitations and Future Work}

Our stimuli temporally varied in $C^{*}$ and $h$, keeping the luminance constant. For the design of these stimuli, we kept the luminance constant for an averaged observer. It is, however, generally known that luminance perception varies over individuals, and consequently, our stimuli might not have been fully isoluminant for all our participants. Nonetheless, we estimate the effect of individually tuned isoluminance to be small for this particular experiment. First of all, various researchers have found that adults are more sensitive to chromatic than luminance contrast for temporal frequencies below $4-5 \mathrm{~Hz}$ [22]. In this experiment specifically, the frequency of the temporal transition was about $0.33 \mathrm{~Hz}$, and at this frequency the data of [22] indicate that adults are at least four times more sensitive to chromatic change than to luminance change. Second, if we simulate possible variations in the $L+M$ channel of our stimuli using the CIE 2006 Physiological Observer (CIEPO06) [23], we found that these variations have a slope close to zero, and at least five times smaller than the slope in any of the chromatic channels [i.e., either $L-M$ or $S-(L+M)$ ]. Still, in our next experiments to extend our data, we will evaluate the effect of including individual isoluminance in the design of our stimuli.

In the current experimental design, lightness changes were not included. Since our long-term goal is to develop a temporal uniform color space, comparing the perceived speed of lightness change with chromaticity change is important. In addition, as mentioned before we omitted the $C C$-comparisons in this study, but they clearly are relevant to extend the set of stimuli for the modeling or to validate the improved color space for temporal color transitions. In the future, lightness changes and more chroma levels will also be included in the experimental design.

Since the task of comparing multiple pairs of sequential color transitions is quite time-consuming, the number of repetitions per participants had to be limited and we were not able to fit a psychometric function per participant. In contrast, the responses were averaged over participants to fit one psychometric function for each hue in the $\mathrm{CH}$ comparisons and each hue in the $H H$ comparisons. We already know that fitting a psychophysical curve across observers may introduce betweenobserver noise. Thus, if time would allow, an ideal case would be fitting a psychometric function per observer. This would require a more efficient psychophysical method or a reduction in the number of experimental conditions, where the latter is undesirable as well.

We intentionally chose for full room setting instead of the often-used $2^{\circ}$ or $10^{\circ}$ stimuli because temporal dynamics in artificial lighting are more realistically represented with a room-size field of view. At the same time, this choice introduces uncertainty about the direction of the observer's gaze and the effects of peripheral vision. For the observers sitting at the predefined position, our stimuli resulted in a $101^{\circ}$ horizontal and roughly $77^{\circ}$ vertical visual angle. Research has shown that both spatial vision [24] and temporal vision [25] vary across this eccentricity, but our data does not allow a comparison of temporal color perception in central or peripheral vision. However, in lighting applications, central and peripheral vision will always mix, so a generally suitable temporal color space is 
needed. Creating models that take eccentricity into account would require new data in the future.

We chose to use a wall illumination of $4000 \mathrm{~K}$ as an intermediate light in an attempt to continuously reset the participants' state of adaptation. Still, participants constantly adapted during the experiment to the varying light environment they were immersed in. This may have affected the experimental results, though most probably in a systematic way. This surely impacts the modeling, since color spaces assume adaption to a fixed illuminant. Due to the lack of a good model for temporal chromatic adaptation (which would change $L, M$, and $S$ reference over time), the calculation of the cone responses to model speed perception can be further improved.

Finally, it should be noted that we might have accumulated error in our calculations to compare the perceived speed of various color transitions with one fixed reference. The accumulated error can be best estimated from the first row of the result summary in Eq. (7). In our experiment, we directly measured that the transition speed along the chroma direction at $5 \mathrm{R}$ needed to be $7.76 \Delta E_{a b}^{*} / s$ in order to be perceived as fast as a transition speed of $10 \Delta E_{a b}^{*} / s$ along the hue direction at $5 \mathrm{R}$. Using the calculation towards the same reference stimulus, we estimate a transition speed of $8.73 \Delta E_{a b}^{*} / s$ along the chroma direction at $5 \mathrm{R}$ to be perceived as fast as a transition speed of 10 $\Delta E_{a b}^{*} / s$ along the hue direction at $5 \mathrm{R}$. The deviation results from the fact that the $H H$ comparison with both the comparison and reference stimulus at $5 \mathrm{R}$ yielded a PSE of 11.25 instead of 10 $\Delta E_{a b}^{*} / s$. It's worth pointing out that $\Delta a^{*} / s$ and $\Delta b^{*} / s$ are linear for $C^{*}$ transitions, but sinusoidal for $h$ transitions, which also has a drawback in the calculation of the slopes according to Fig. 8.

Based on the just-mentioned limitations, we see multiple options for further research. Perception of dynamic color has many dimensions, such as three dimensions to define a base point in a color space, three dimensions of the modulation direction, and the related periodicity and waveform. In the future, more base colors (hues) and modulation directions (not only cardinal directions) will be needed to more accurately model a temporally uniform color space. Instead of defining the related stimuli in CIELAB, we see added value in defining them directly in cone-contrast space. In addition, there are more ways of generating the shape of the stimuli. Rather than giving all stimuli a fixed time period and changing the speed by changing the amplitude of the chroma or hue, one could keep the chroma or hue amplitude constant and change the speed by changing the time interval. The disadvantage of this choice, though, may be that observers use the additional time cue to judge the perceived speed of the color transition. Finally, it could be advantageous to use different JNDs instead of PSEs to distinguish perceived speeds, so they can be further used to build the temporal sensitivity function.

\section{CONCLUSIONS}

In this study, the perceived speed of periodic color transitions in chroma and hue was measured, starting from the five principal Munsell hues. The results indicate that available color spaces are not able to predict our speed perception. It is not so surprising that CIELAB is not perceptually uniform for temporal color transitions, as it was designed for describing spatial color differences. Color spaces built on cone responses were also insufficiently accurate to predict uniform speed perception of color transitions. Simple modifications of these spaces improve the uniformity in speed perception, but we foresee more extensive experimentation to build a more accurate model.

Funding. China Scholarship Council (CSC) (201506950017); Wuhan University of Technology (WUT) (104971140150).

Acknowledgment. We thank the staff and students of Rochester Institute of Technology for their participation in the experiments. We also would like to thank the three anonymous reviewers whose comments/suggestions helped improve and clarify this paper.

\section{REFERENCES}

1. W. van Bommel, "Dynamic lighting at work-both in level and colour," in 2nd CIE Expert Symposium on Light and Health, CIE X031:2006 (2006), pp. 62-67.

2. I. Vogels, D. Sekulovski, and B. Rijs, "How to create appealing temporal color transitions?" J. Soc. Inf. Disp. 17, 23-28 (2009).

3. M. Hartog, "Dynamic coloured lighting: an interaction concept for the creation and control of atmospheres at home," Master's thesis (Delft University of Technology, 2010).

4. M. Murdoch, D. Sekulovski, and P. Seuntiëns, "The influence of speed and amplitude on visibility and perceived subtlety of dynamic light," in Color and Imaging Conference (2011), pp. 265-269.

5. B. Li, Q. Y. Zhai, J. B. Hutchings, M. R. Luo, and F. T. Ying, "Atmosphere perception of dynamic LED lighting over different hue ranges," Light. Res. Technol. (to be published).

6. B. A. Salters and M. P. C. M. Krijn, "Color reproduction for LED-based general lighting," Proc. SPIE 6338, 63380F (2006).

7. D. Sekulovski, I. M. Vogels, M. Van Beurden, and R. Clout, "Smoothness and flicker perception of temporal color transitions," in Color and Imaging Conference (2007), pp. 112-117.

8. D. Sekulovski, I. Vogels, R. Clout, and M. Perz, "Changing color over time," in Ergonomics and Health Aspects of Work with Computers, M. M. Robertson, ed. (Springer, 2011), Vol. 6779 LNCS, pp. 218-225.

9. R. G. Kuehni, "Hue uniformity and the CIELAB space and color difference formula," Color Res. Appl. 23, 314-322 (1998).

10. H. Knau, "Thresholds for detecting slowly changing Ganzfeld luminances,” J. Opt. Soc. Am. A 17, 1382-1387 (2000).

11. A. M. Derrington, J. Krauskopf, and P. Lennie, "Chromatic mechanisms in lateral geniculate nucleus of macaque," J. Physiol. 357, 241-265 (1984)

12. M. D. Fairchild, Color Appearance Models (Wiley, 2013).

13. M. J. Murdoch, "Characterization and control of a multi-primary LED light lab," Opt. Express 25, 29605-29616 (2017).

14. M. Hauta-Kasari, "Munsell colors matt (Spectrofotometer measured)," 1999 [retrieved November 14, 2017], https://www.uef.fi/en/web/ spectral/munsell-colors-matt-spectrofotometer-measured.

15. O. Rinner and K. R. Gegenfurtner, "Time course of chromatic adaptation for color appearance and discrimination," Vision Res. 40, 1813-1826 (2000).

16. M. Shuttleworth, Counterbalanced Measures Design (2009).

17. F. A. Wichmann and N. J. Hill, "The psychometric function: II. Bootstrap-based confidence intervals and sampling," Percept. Psychophys. 63, 1314-1329 (2001).

18. A. Stockman and L. T. Sharpe, "The spectral sensitivities of the middle- and long-wavelength-sensitive cones derived from measurements in observers of known genotype," Vision Res. 40, 1711-1737 (2000). 
19. R. F. Dougherty, W. A. Press, and B. A. Wandell, "Perceived speed of colored stimuli," Neuron 24, 893-899 (1999).

20. D. Nguyen-Tri and J. Faubert, "The perceived speed of drifting chromatic gratings is mechanism-dependent," Vision Res. 42, 2073-2079 (2002).

21. D. J. McKeefry and M. P. Burton, "The perception of speed based on L-M and S-(L+M) cone opponent processing," Vision Res. 49, 870-876 (2009).

22. K. R. Dobkins, C. M. Anderson, and B. Lia, "Infant temporal contrast sensitivity functions (tCSFs) mature earlier for luminance than for chromatic stimuli: evidence for precocious magnocellular development?" Vision Res. 39, 3223-3239 (1999).

23. Comission Internationale de L'Eclairage (CIE), "Fundamental chromaticity diagram with physiological axes," CIE Technical Report (2005).

24. T. Hansen, L. Pracejus, and K. R. Gegenfurtner, "Color perception in the intermediate periphery of the visual field," J. Vis. 9(4):26 (2009).

25. M. Perz, D. Sekulovski, and M. Murdoch, "Chromatic flicker perception in human peripheral vision under mental load," in Color and Imaging Conference (2010), pp. 33-37. 